\title{
ADHERENCE TO TREATMENT ASSESSMENT IN PATIENTS WITH CARDIOVASCULAR DISEASES FROM A CLINICAL AND PSYCHOLOGICAL PERSPECTIVE
}

\author{
Maria Iakovleva \\ Department Medical Psychology and Psychophysiology, Saint Petersburg State University (Russia)
}

\begin{abstract}
Introduction. The behavior of patients with cardiovascular diseases in the course of therapy is of key importance, since their survival and quality of life depend on their lifestyle and adherence to physicians' recommendations. Adherence to treatment and its assessment is considered to be a medical problem, but numerous studies show that it is multifactorial and it must be considered from an interdisciplinary point of view.

Material and Methods. CHD patients of the cardiac rehabilitation department were examined $(n=112)$. Patients' behavior in the therapy process, their clinical, emotional and social status and psychological characteristics were studied. Supervising doctors and clinical psychologists who followed up the patients made an expert opinion on their level of adherence to treatment and divided them into two groups. At the same time, they filled out a specific questionnaire for each patient, which contained several aspects of patients' therapeutic behavior, both pharmacological and non-pharmacological.

Results. Adherent and non-adherent patients were found to differ in all the aspects included in the questionnaire $(p<0,001 ; p<0,05$ for the indicator "alcohol consumption"). The factor analysis of all the questions from the questionnaire identified three major factors that combined various aspects of treatment adherence. Three obtained factors explain $70 \%$ of the total dispersion. The $1^{\text {st }}$ one included the following aspects: regularity of cardiological examinations, regularity of blood pressure self-monitoring, intake of prescribed medications regularity; the $2^{\text {nd }}$, control (and, if necessary, reduction) of weight, following a diet, the level of physical activity; the $3^{\text {rd }}$, alcohol consumption (frequency and dose) and smoking (smoking cessation/number of cigarettes per day). Based on the three identified factors system, patients can be divided into more specific categories.

Conclusion. Nevertheless, it is essential to consider not only the level of adherence to treatment and its aspects, but also the reasons of patients' behavior: their awareness of the treatment regimen, their consciousness and motives for following the recommendations, the value of health and the subjective disease perception. It is important to take account of the factors that may interfere in adherence to treatment, and patients' resources for an optimal therapeutic behavior. Therefore, adherence to treatment is based on both clinical and psychological aspects. Its theoretical and practical study should be based on the biopsychosocial approach to the problem and an individual approach to each patient.
\end{abstract}

Acknowledgement. This research was supported by the Russian Foundation for Basic Research (RFBR) (Grant No 18-013-00689 A).

Keywords: Therapeutic behavior, adherence to treatment, assessment, cardiovascular diseases.

\section{Introduction}

The problem of adherence to treatment is relevant primarily for chronic diseases: according to the WHO the rates of non-adherence are about $50 \%$ (WHO, 2003). At the same time, a low level of adherence to treatment presents a menace both to the patient's health condition (for example, in cases of diabetes, coronary heart disease, etc.), and to others' health (in cases of tuberculosis, HIV, etc.).

It is also noted that patients' adherence to treatment is significant for the healthcare system: low adherence leads to repeated manifestations of the disease and increases the cost of treatment, as well as leads to a decrease of trust in the doctors due to poor treatment results, undermines the belief in medicine among patients and their families. 
The behavior of patients with cardiac diseases in the treatment process is of key importance, since their survival and quality of life depend on their lifestyle and following the doctors' recommendations on pharmacotherapy. Nevertheless, to date, adherence to treatment among such patients remains low, and mortality rates for this group of diseases are leading.

A high level of adherence to treatment is also crucial after cardiosurgical interventions: the duration of the positive effect of such operations as heart transplantation, myocardial revascularization (coronary stenting and bypass surgery) depends on how accurately the patient will comply with the cardiologists' recommendations.

In connection with the particular urgency of the patients' adherence to medical recommendations problem, the question of its assessment becomes actual. In practical medicine, adherence to treatment is most often assessed in a conversation with the patient. Any doubt about the sincerity of the patient is resolved by questioning relatives and/or (less commonly) additional diagnostic procedures and tests. Patients' regular visits to the check-ups, their active participation in the discussion of treatment issues, trustful attitude indicate a higher level of adherence (Konradi A.O., 2007). The most commonly used methods aim at measuring pharmacological adherence (Fofanova T.V., 2015). However, adherence to treatment for coronary heart disease (CHD) is not limited to adherence to pharmacotherapy, but includes a whole range of aspects - constant monitoring of blood pressure, healthy nutrition, recommended physical activity, etc., which are associated with the specifics of the disease. It is worth mentioning that in terms of adherence to non-drug therapy, a single criterion of adherence has not yet been developed (Jin J. et al., 2008). The existing clinical methods, as well as self-report methods, indicate that this problem is of a medical nature. However, in practice, the task of assessing the level of adherence is often assigned to the clinical psychologist who treats the patient at the pre- and postoperative stages.

\section{Material and methods}

Objective: to study the behavior of patients in the therapy process - their adherence to the recommendations regarding pharmacological and non-pharmacological treatment, lifestyle changes; and to study the possibility of using a multifactorial model in the assessment of the level of patients' adherence to treatment taking into account various behavioral aspects.

The present study is part of a larger research, which results show the great role of the patients' psychological characteristics (in conjunction with social and clinical characteristics) in the rehabilitation process after surgical treatment of coronary heart disease (Iakovleva M. et al., 2019). The research is being held at the St. Petersburg State University and on the basis of the Almazov National Medical Research Centre (Saint Petersburg), from 2012 to the present time.

A survey of 112 patients undergoing treatment in the cardiology department is presented. All examined patients were diagnosed with CHD, they underwent direct myocardial revascularization; no mental pathology or cognitive decline was found. $81 \%$ were men; 58,4 years was the sample average age.

The assignment of a patient to a group of adherent or non-adherent to treatment was made by an expert method, jointly by a cardiologist and a clinical psychologist. At the same time, for each patient a questionnaire was filled out, taking into account many behavioral factors associated with adherent behavior. In total, it contained 8 questions (indicators) (see Figure 1), each of them could be rated at 0,1 , or 2 points. Higher scores indicated a higher level of adherence. Further analysis of the questionnaire showed that all the aspects indicated in it are significant for determining adherence to therapy and should be taken into account when assessing it.

\section{Results}

Data obtained using the adherence to treatment questionnaire was analyzed in the study. Indicators on each of the 8 scales included in the questionnaire (i.e. aspects of adherence) for the two groups of patients are presented in Figure 1. 
Figure 1. Mean values in the groups of adherent and non-adherent to treatment patients according to different aspects of treatment adherence.

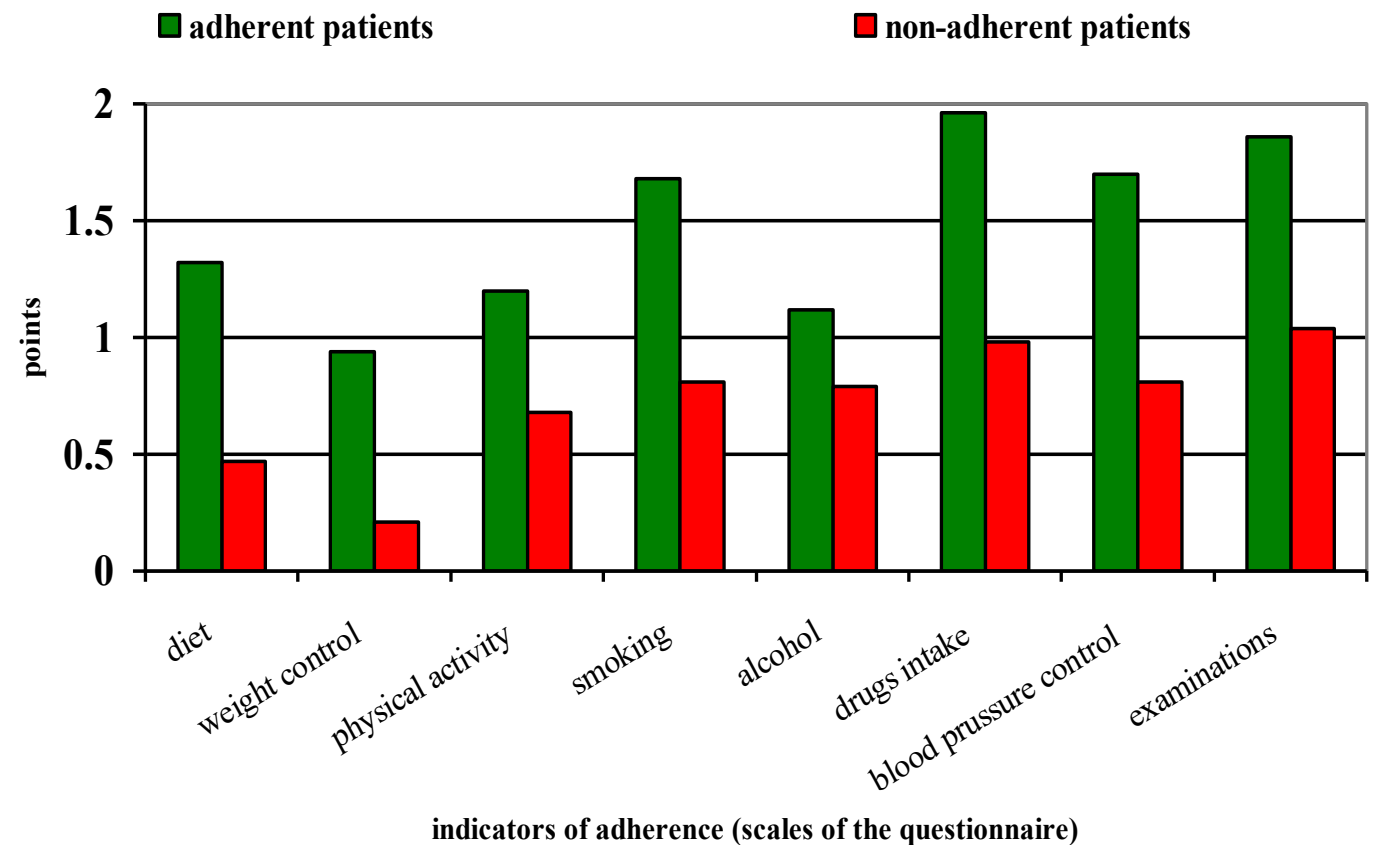

Note. The minimum number of points that a patient could score on each of the scales is 0 (correspond to the pole of non-adherence to treatment); maximum - 2 (correspond to the pole of adherence to treatment).

Comparison of two groups of patients according to various indicators included in the adherence to treatment questionnaire revealed that patients differ in almost all aspects of the questionnaire: following a diet, weight control, physical activity, smoking, medication intake, blood pressure self-monitoring, regularity of medical examinations $(p<0.001)$. The groups also differ in terms of "alcohol consumption" $(p<0.05)$. Thus, patients assigned to the group of adherents to treatment significantly gain more points on all scales of the questionnaire, i.e. they have higher indicators of adherence, which may say for the effectiveness of the questionnaire.

Next, the whole set of questions on the adherence to treatment questionnaire, i.e., various aspects and indicators of the phenomenon of adherence, was subjected to factor analysis using the principal component method.

As a result of varimax rotation with Kaiser normalization, three major factors that combined various aspects of treatment adherence were revealed. The content of three factors obtained, i.e., which parameters are included in a particular factor and what is their tese coefficient (factor weight), can be identified based on the data of mathematical analysis (Table 1). These three factors account for $70 \%$ of the total variance of characters.

Table 1. The results of factor analysis of various adherence aspects (factor weights and factorization).

\begin{tabular}{|l|r|r|r|}
\hline \multirow{2}{*}{ Aspects of adherence to treatment } & \multicolumn{3}{|c|}{ Component } \\
\cline { 2 - 4 } & 1 & \multicolumn{1}{|c|}{2} & 3 \\
\hline Check-ups & 0,878 & & \\
\hline Blood pressure control & 0,819 & & \\
\hline Intake of prescribed medications & 0,788 & & \\
\hline Weight control & & 0,841 & \\
\hline Diet & & 0,802 & \\
\hline Physical activity & 0,434 & 0,511 & \\
\hline Alcohol consumption & & & 0,765 \\
\hline Smoking & & & 0,660 \\
\hline
\end{tabular}

Factor 1. This factor included the following indicators (aspects) of adherence to treatment: regularity of medical examinations; regularity of blood pressure self-monitoring; regularity of prescribed medications intake (dosages and regimen). The informative value of this factor is $30 \%$; it could be designated as "medical". 
Factor 2. It included the following indicators: patients' control (and decrease, if necessary) of weight; following a diet (restrictions on various groups of products); level of physical activity, regularity of physical exercise. The informative value of the factor is $23 \%$; it could be called "physical condition".

Factor 3. It included the following two indicators: alcohol consumption (frequency and dose) and smoking (recommended smoking cessation / frequency and number of cigarettes smoked). The informative value of the factor is $17 \%$; it could be called "bad habits".

All indicators included in the abovementioned factors have a positive factor weight: the positive pole of the factor is interpreted by the positive poles of the variables included in it, that is, a larger value (and therefore, a better result) is obtained with large points scored for these variables.

\section{Discussion}

Patient samples can be divided into more specific categories based on the three identified factors. So, for example, a group of patients who regularly seek for medical help, take medications, but who have not abandoned bad habits (i.e., taking into account the 1st and 3rd factors), or a group of patients who do not consume alcohol and tobacco, but refuse pharmacotherapy, can be distinguished. Patients from these groups may differ in their psychological characteristics, in motivation for continuing treatment and health beliefs; therefore, it is more effective considering them, as well as developing psychocorrectional programs for them, taking into account these differences, and not according just to one isolated quantitative indicator of adherence to treatment.

\section{Conclusions}

Adherence to treatment is usually considered a medical problem. Thus, recommendations in case of patient's non-adherence are also of a medical nature. However, numerous studies show that this is a complex multifactorial problem, and its consideration seems more productive from an interdisciplinary point of view. Adherence to treatment for most chronic diseases is not limited to adherence to drug therapy, but includes a whole range of aspects related to a lifestyle change, specific to the illness.

Summarizing the above, adherence to treatment is a complex phenomenon associated not only with the patient's behavior in the therapy process, but also with his/her attitudes, level of awareness, health literacy, previous medical experience, the influence of relatives, the relationship with the doctor and the perception his/her disease. It is important to take account of the factors that may interfere in adherence to treatment, and patients' resources for an optimal therapeutic behavior. Therefore, adherence to treatment is based on both clinical and psychological aspects. Its theoretical and practical study should be based on the biopsychosocial approach to the problem and an individual approach to each patient.

\section{Acknowledgements}

This research was supported by the Russian Foundation for Basic Research (RFBR) (Grant No 18-013-00689 A).

\section{References}

Fofanova, T.V. (2015). Adherence to therapy and methods for increasing it in patients with arterial hypertension and coronary heart disease (Doctoral dissertation). Retrieved from: https://cardioweb.ru/files/autoref/191/avtoref.pdf.

Iakovleva, M., Kruglova, N., Shchelkova, O., Lubinskaya, E., \& Nikolaeva, O. (2019). Potentials of psychological prediction of patients' therapeutic behavior after myocardial revascularization. C. Pracana \& M. Wang (Eds.), Psychological Applications and Trends 2019 (10-13). Lisboa: InScience Press.

Jin, J., Sklar, G. E., Min Sen Oh, V., \& Chuen Li, S. (2008). Factors affecting therapeutic compliance: A review from the patient's perspective. Therapeutics and Clinical Risk Management, 4(1), 269-286.

Konradi, A.O. (2007). The importance of adherence to therapy in the treatment of cardiac diseases. Spravochnik poliklinicheskogo vracha, 4(6), 8-11.

World Health Organisation. (2003). Adherence to long-term therapies, evidence for action. Geneva: WHO. 